\title{
Size Distribution, Imaging and Growth Mechanisms of Self-Assembled InAs Quantum Wires on Vicinal Substrates
}

\author{
A Scullion $^{1}$, D A Thompson ${ }^{2}$ and G A Botton ${ }^{1}$ \\ 1. Department of Materials Science and Engineering, McMaster University, Hamilton, Canada \\ 2. Department of Engineering Physics and Centre for Emerging Devices Technology, McMaster \\ University, Hamilton, Canada
}

Quantum well structures provide us with the ability to tailor the electronic and photoluminescent properties of semiconductor materials for various applications such as solar cells, diodes and lasers. 2dimensional quantum wells are now relatively easy to produce by depositing multiple lattice-matched semiconductor layers onto a substrate using chemical or physical deposition techniques. Molecular beam epitaxy (MBE) has the advantage that it can produce abrupt interfaces with atomic monolayer precision. Despite having a sharp interface, the spectral line width of these devices is still limited by the stepwise density of states produced by confinement in only one dimension. This problem can potentially be overcome by fabricating lower-dimensional structures such as quantum wires and quantum dots.

Due to the limitations of electron beam lithography in terms of spatial resolution as well as scalability, self-assembled quantum structures have been under thorough investigation over the past couple of decades with a fair amount of success [1]. Material deposited onto a lattice-mismatched substrate undergoes a strain-relief mechanism known as the Stranski-Krastanov growth mode where it forms islands. Given that the deposited material's bandgap is smaller than that of the substrate, isotropic islands form quantum dots and elongated islands form quantum wires. The challenge involving self-assembled quantum wires is to produce them with a narrow size distribution as their size influences their luminescence spectra. The unevenness of self-assembled quantum structures has so far limited their performance in terms of photoluminescent line width for use in laser devices.

This work aims to study the effect of growing quantum wires on a vicinal (off-cut) substrate as well as the effect of growth conditions such as temperature and barrier layer composition in an effort to produce wires with a narrower size distribution. Growth on a vicinal substrate has been shown to be beneficial in some studies [2] but not in others [3]. Figure 1a shows wires grown on $\operatorname{In}_{.53} \mathrm{Ga}_{37} \mathrm{Al}_{10} \mathrm{As}$ lattice-matched to $\operatorname{InP}(001)$ off-cut by $0.9^{\circ}$ towards the (110) direction. This angle was chosen in the hope that the atomic steps will serve as a template for the quantum wires to grow on. At this particular angle, the atomic step length corresponds to the average distance between the wires grown on a nominal substrate and there is evidence that quantum wires tend to nucleate at step edges [4].

The micrograph is a secondary electron image obtained with a scanning electron microscope (SEM) (FEI Magellan 400) with an in-lens detector using a landing energy of $1 \mathrm{keV}$. Due to the very thin geometry of the wires $(\sim 2 \mathrm{~nm}$ peak to trough) and the relatively large electron interaction volume, the typical gradient-based topographical contrast is not present here. Instead the brightest region is the top of the wires since this area allows, presumably, for secondary electrons to escape on either side of the wires. It is also possible that an increased valence electron density may also have been measured [5]. This microstructure is similar to the height profile from a kinetic Monte Carlo (kMC) simulation (figure 1b) of strained adatom diffusion which is currently being developed to better understand the formation of quantum dots and wires. Some of the important characteristics of the wires present in both images are their meandering shape and slight variations in height along them. 
Figure 2 shows a high resolution scanning transmission electron microscope (HRSTEM) high-angle annular dark-field (HAADF) image of a cross-sectional sample of InAs quantum wires, obtained with an FEI Titan 80-300 equipped with an aberration corrector of the probe-forming lens. The yellow line indicates a (001) plane through the sample illustrating the $0.9^{\circ}$ off-cut expected from the wafer preparation. Images such as these provide the ideal means to locate the position of the atomic steps with respect to the wires and assess the role of steps in nucleation. In this case, we can see additional features above the wires consistent with local segregation of indium during growth. Further analysis regarding the size distribution of wires grown in various conditions will be discussed [6].

\section{References:}

[1] R Noetzel, Semiconductor Science and Technology 11 (1996), p. 1365.

[2] B Salem et al, Applied Physics Letters 79 (2001), p. 4435.

[3] W Lei et al, Applied Physics Letters 90 (2007), p. 103118.

[4] S I Molina et al, Applied Physics Letters 91 (2007), p.143112.

[5] K Cui et al, Journal of Physics: Conference Series 326 (2011), p. 12007.

[6] This work was carried out at the Canadian Centre for Electron Microscopy, a national facility supported by NSERC and McMaster University.
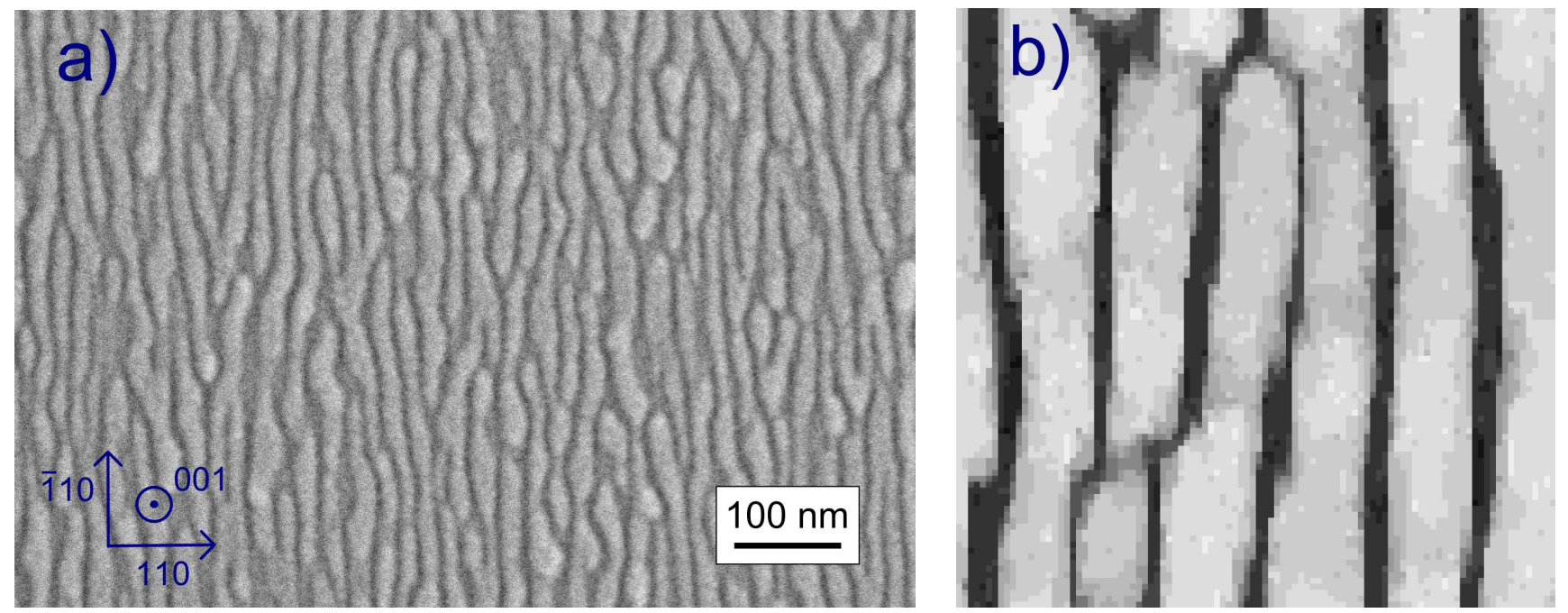

Figure 1. a) SEM micrograph of InAs quantum wires. b) kMC simulation of adatom diffusion producing elongated islands. The simulated area is 100x100 atoms.

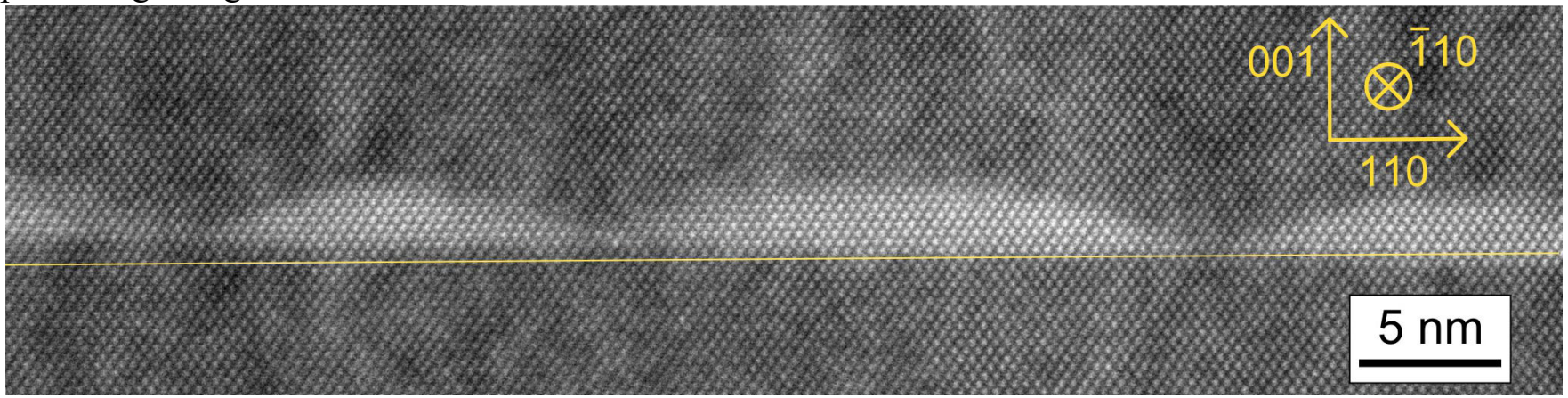

Figure 2. HRSTEM-HAADF cross-sectional micrograph of quantum wires along the (-110) direction. The yellow line indicates a (001) plane. 